\title{
Study of neutrophil lymphocyte ratio (NLR) and platelet lymphocyte ratio (PLR) as a predictor inflammatory marker for diabetic nephropathy in type 2 diabetic patients
}

\author{
Ahmed Ateia Abdelaziz ${ }^{1}$; El-Sayed El-Meghawry El-Sayed ${ }^{2}$; Tarek Mustafa Emran ${ }^{3}$ and \\ Ali Ibrahim Abdallah ${ }^{2}$ \\ ${ }^{1}$ DepartmentofInternal Medicine; Faculty of Medicine, Al-Azhar University (Cairo), ${ }^{2}$ Department of Internal Medicine; \\ ${ }^{3}$ Department of Clinical Pathology; Damietta Faculty of Medicine, Al-Azhar University \\ Corresponding author: Ali Ibrahim Abdallah, E-Mail: alilonly6@gmail.com, Mobile: 01012466203
}

\begin{abstract}
Background: Diabetes mellitus (DM) is a systemic disease having serious microvascular and macrovascular complications. DN in T2DM has an inflammatory pathology. Many inflammatory markers have been found to be related to DN, such as interleukin1 (IL1), IL6, IL8, transforming growth factor beta 1(TGF- $\beta 1$ ) and tumor necrosis factor alpha $(\mathrm{TNF} \alpha)$. However, their measurement is not used routinely as it is not easy to do it, in this respect. Search for inflammatory markers for the disease is a continuous process to enhance the diagnostic and treatment process.

Aim of the Work: To assess neutrophil lymphocyte ratio (NLR) and platelet lymphocyte ratio (PLR) as a predictor inflammatory markers for diabetic nephropathy in type 2 diabetic patients.

Patients and Methods: This study is a prospective one that was carried out on one hundred (100) type 2 Diabetes mellitus (T2DM) patients attending to internal medicine outpatient clinic and inpatient department of internal medicine at Al-Azhar university hospital, Damietta and twenty five (25) apparently healthy volunteers as a control. The populations of the study were classified into three groups according to their level of albuminuria. All were subjected tofull history and Clinical examination, Laboratory tests include Fasting mid-stream urine samples were obtained and examined for complete urine analysis and albumin/creatinine ratio (UACR),Complete blood count (CBC), HbA1c,Fasting blood sugar, Renal Function Tests,eGFR and Abdominal ultrasonography, Fundus examination and ECG and Assessment of NLR \&PLR.

Results: Our study showed that there was high statistically significant increased NLR, PLR and UACR in group IB when compared to group IA, II and III. Also there was statistically significant increased NLR,PLR and UACR in group IA in comparison to group II and also there was statistically significant increased NLR,PLR and UACR in group II in comparison to group III.

Conclusion: Neutrophil lymphocyte ratio (NLR) \& Platelet lymphocyte ratio (PLR) was significantly associated with diabetic nephropathy (DN) and high Neutrophil lymphocyte ratio (NLR) \& Platelet lymphocyte ratio (PLR) values may be considered as a predictor and a prognostic risk markers of diabetic nephropathy (DN).
\end{abstract}

Keywords: Albuminuria; diabetic nephropathy; inflammation; neutrophil/lymphocyte ratio; platets/lymphocyte ratio

\section{INTRODUCTION}

Diabetes mellitus (DM) is a systemic disease having serious microvascular and macrovascular complications. Microvascular complications include diabetic nephropathy (DN), diabetic retinopathy, and diabetic neuropathy while macrovascular complications include stroke, cardiovascular diseases (CVDs), and peripheral vascular diseases ${ }^{(1)}$. Diabetic nephropathy (DN) is a clinical syndrome characterized by persistent albuminuria ( $>300 \mathrm{mg} / 24$ $\mathrm{hr}$, or $300 \mathrm{mg} / \mathrm{g}$ creatinine), a progressive decline in glomerular filtration rate (GFR), arterial hypertension, increased cardiovascular morbidity and mortality and eventually end stage renal disease (ESRD) ${ }^{(2)}$. The degree of albuminuria is not necessarily linked to disease progression in patients with DN associated with either type 1 or type 2 diabetes mellitus (T2DM) $)^{(3)}$.

Diabetic nephropathy affects approximately $25 \%$ of patients with T2DM and represents the leading cause of ESRD ${ }^{(4) .}$
Rudiger et al. ${ }^{(5)}$ showed that the relationship between systemic inflammation and vascular disease indicated that chronic inflammation promotes the development and acceleration of micro and macro angiopathic complications in patients with diabetes. Also Rudiger et $\boldsymbol{a l}^{(5)}$ confirmed that Total white blood cell (TWBC) count is a crude but sensitive indicator of inflammation which can be done easily in laboratory routinely, being also a cost effective investigation and showing increase in the neutrophil count in thrombus formation and ischemic diseases. The neutrophil lymphocyte ratio (NLR) in complete blood count is studied in many cardiac and noncardiac diseases as an inflammatory marker and is used to predict the prognosis of diseases.

White blood cell and differential count can be done as a part of routine automated hematology analyzer. Recently, neutrophil lymphocyte ratio (NLR) has been evaluated and used as inflammatory marker in malignancies, infection and coronary artery diseases ${ }^{(6)}$. In addition Celikbilek et al. ${ }^{(7)}$ observed that Neutrophil/lymphocyte ratio (NLR) possesses a 
diagnostic value in certain pathologies characterized by systemic or local inflammatory response such as diabetes mellitus, coronary artery disease, ulcerative colitis and inflammatory arthritis.

DN in T2DM has an inflammatory pathology. Many inflammatory markers have been found to be related to DN, such as interleukin1 (IL1), IL6, IL8, transforming growth factor beta 1(TGF- $\beta 1$ ) and tumor necrosis factor alpha (TNF $\alpha$ ). However, their measurement is not used routinely as it is not easy to do it, in this respect; NLR has emerged as a novel surrogate marker ${ }^{(8) .}$

Turkmen et al. ${ }^{(9)}$ showed that platelets can interact with various cell types, including endothelial cells, T-lymphocytes, neutrophils and mononuclear phagocytes, in which earlier investigations strongly suggested that chronic inflammation, may contribute to the development of atherosclerosis. Beside that platelet lymphocyte ratio (PLR) was found to be higher in Rheumatoid arthritis (RA) patients compared to healthy controls.

Amaylia et $\boldsymbol{a l} .{ }^{(6)}$ found that NLR was significantly higher in SLE than normal subjects.

Sagar et $\boldsymbol{a l}^{(10)}$ found that there was a significant correlation between NLR and DN, implying that inflammation and endothelial dysfunction could be an integral part of DN.

So, it is of value for early predictors of DN by which we can predict the disease and can halt the progression of the disease through routinely done, easy and cost effective markers like NLR and PLR.

\section{AIM OF THE WORK}

The aim of this study is to assess neutrophil lymphocyte ratio (NLR) and platelet lymphocyte ratio (PLR) as a predictor inflammatory markers for diabetic nephropathy in type 2 diabetic patients.

\section{PATIENTS AND METHODS}

This study is a prospective one that was carried out on one hundred (100) type 2 Diabetes mellitus (T2DM) patients attending to internal medicine outpatient clinic and inpatient department of Internal Medicine, Al-Azhar University hospital, Damietta and twenty five (25) apparently healthy volunteers as a control. The populations of the study were classified as follow:

Group I: Fifty(50)Type 2 Diabetes mellitus (T2DM) patients with diabetic nephropathy (DN) divided as follow: I A:Twenty five (25) Type 2DM patients with DN with Microalbumenuria. I B:Twenty five (25) Type 2DM patients with DN with Macroalbumenuria.

Group II: Fifty (50) Type 2DM patients with Normoalbuminuria.

Group III: Twenty five (25) apparently healthy volunteers matched for age and sex as a control.

Exclusion criteria: Patients with type 1 DM. Patients with infections, for example, urinary tract infection (UTI), upper respiratory tract infection, lower respiratory tract infections, gastrointestinal infection, and pyrexia of unknown origin. Patients with systemic disorder such as chronic liver disease, blood disorders, autoimmune disorders. Any hypertensive patients not related to diabetic complications. patients with uncontrolled blood pressure (BP). Pregnant women with T2DM (particularly with preeclampsia). Patients on antiinflammatory drugs, systemic or topical steroids, angiotensin-converting enzyme inhibitors, angiotensin II receptor blockers or alcohol. patients having diseases affecting urinary protein excretion as nephritic syndrome, renal artery stenosis, dehydration state, and UTI; and patients having low glomerular filtration rate (GFR)without microalbuminuria.

Methods: All were subjected to full history and Clinical examination, Laboratory tests include Fasting mid-stream urine samples were obtained and examined for complete urine analysis and albumin/creatinine ratio (UACR), Complete blood count (CBC), HbA1c,Fasting blood sugar, Renal Function Tests, eGFR was calculated using CKDEpidemiology Collaboration (CKD-EPI) formula, Abdominal ultrasonography, Fundus examination and ECG, Evaluation of DN was done by examining urinary albumin/creatinine ratio (UACR) in a spot urine sample. Assessment of NLR by dividing the absolute neutrophil count on the absolute lymphocyte count. Assessment of PLR by the platelet count dividing on the absolute lymphocyte count. An informed consent was taken by each patient before the procedure. The study was approved by the Ethics Board of Al-Azhar University.

Statistical methods: Data were collected, coded, revised and entered to the Statistical Package for Social Science (IBM SPSS) version 20. The data were presented as number and percentages for the qualitative data, mean, standard 
deviations and ranges for the quantitative data with parametric distribution and median with inter quartile range (IQR) for the quantitative data with non parametric distribution. Chi-square test was used in the comparison between two groups with qualitative data and Fisher exact test was used instead of the Chi-square test when the expected count in any cell found less than 5.The comparison between more than two groups with quantitative data and parametric distribution were done by using One Way Analysis of Variance (ANOVA) test and Kruskall-Wallis test was used in the comparison between more than two groups with quantitative data and non-parametric distribution. All tests were two sided. p-value $<0.05$ was considered statistically significant, $\mathrm{p}$-value $<0.001$ was considered highly statistically significant, and pvalue $\geq 0.05$ was considered non statistically significant.

\section{RESULTS}

Table (1): StatisticalComparison between Group IA, IB, II and III as regards CBC

\begin{tabular}{|c|c|c|c|c|c|c|c|c|c|c|c|}
\hline & & \multicolumn{2}{|c|}{ Group I A } & \multicolumn{2}{|c|}{ Group I B } & \multicolumn{2}{|c|}{ Group II } & \multicolumn{2}{|c|}{ Group III } & \multicolumn{2}{|c|}{$\begin{array}{l}\text { One way } \\
\text { ANOVA }\end{array}$} \\
\hline & & Mean & SD & Mean & SD & Mean & SD & Mean & SD & $\mathbf{F}$ & $\begin{array}{c}\mathbf{P} \\
\text { value }\end{array}$ \\
\hline \multicolumn{2}{|c|}{$\begin{array}{l}\text { WBCs } \\
\left(4.0-11.0 \times 10^{3} / \mathrm{ml}\right)\end{array}$} & 5.02 & 1.31 & 5.76 & 1.07 & 5.26 & 1.54 & 5.97 & 0.80 & 3.194 & 0,026 \\
\hline \multicolumn{2}{|c|}{$\begin{array}{l}\mathrm{HB} \\
(12-16 \mathrm{~g} / \mathrm{dL} ? 13- \\
17 \mathrm{~g} / \mathrm{dL})\end{array}$} & 10.34 & 1.44 & 11.44 & 1.21 & 11.04 & 1.30 & 12.48 & 1.24 & 12.238 & $<0.001$ \\
\hline \multicolumn{2}{|c|}{$\begin{array}{l}\text { PLT } \\
150-450 \times\left(10^{3}\right) / \mathrm{ml}\end{array}$} & 257.44 & 69.88 & 290.24 & 54.08 & 287.08 & 54.26 & 262.96 & 55.90 & 2.376 & 0,073 \\
\hline \multicolumn{2}{|c|}{$\begin{array}{l}\text { Neutrophil } \\
\left(2.0-7.5 \times 10^{3} / \mathrm{ml}\right)\end{array}$} & 3.14 & 0.83 & 4.02 & 0.88 & 3.20 & 0.89 & 3.19 & 0.58 & 6.899 & $<0.001$ \\
\hline \multicolumn{2}{|c|}{$\begin{array}{l}\text { Lymphocyte } \\
\left(1.5 .-4.5 \times 10^{3} / \mathrm{ml}\right)\end{array}$} & 1.11 & 0.36 & 1.14 & 0.34 & 1.75 & 0.53 & 2.54 & 0.48 & 54.507 & $<0.001$ \\
\hline \multicolumn{12}{|c|}{ Post hoc test } \\
\hline & \multicolumn{3}{|c|}{$\begin{array}{c}\text { Group IA VS Group } \\
\text { IB }\end{array}$} & \multicolumn{4}{|c|}{ Group IA VS Group II } & \multicolumn{4}{|c|}{ Group II VS Group III } \\
\hline WBCs & \multicolumn{3}{|c|}{0.042} & \multicolumn{4}{|c|}{0.444} & \multicolumn{4}{|c|}{0.025} \\
\hline $\mathrm{HB}$ & \multicolumn{3}{|c|}{0.003} & \multicolumn{4}{|c|}{0.031} & \multicolumn{4}{|c|}{$<0.001$} \\
\hline PLT & \multirow{2}{*}{\multicolumn{3}{|c|}{0.048}} & \multirow{2}{*}{\multicolumn{4}{|c|}{$\begin{array}{ll}0.039 \\
0.782\end{array}$}} & \multicolumn{4}{|c|}{0.092} \\
\hline Neutrophil & $<0.001$ & & & & & & & \multicolumn{4}{|c|}{0.968} \\
\hline $\begin{array}{l}\text { Lymphocy } \\
\text { te }\end{array}$ & \multicolumn{3}{|c|}{0.853} & \multicolumn{4}{|c|}{$\begin{array}{l}0.782 \\
<0.001\end{array}$} & \multicolumn{4}{|c|}{$<0.001$} \\
\hline
\end{tabular}

Our study showed that there is high statistically significant increase as regard neutrophils count $/ \mathrm{mm} 3$ in group IB in comparison to group IA, II and III. There was statistically significant decrease as regard Lymphocytes count $/ \mathrm{mm} 3$ in group IB in comparison to group IA, II and III. In group IA in comparison to group II and III. and in group II in comparison to group III. There is statistically significant increase as regard Platelets count $/ \mathrm{mm} 3$ in group IB in comparison to group IA, II and III. In group IA in comparison to group II and III.
Table (2): Statistical comparison between Group IA, IB, II and III as regards NLR, PLR and UACR.

\begin{tabular}{|c|c|c|c|c|c|c|c|c|c|c|}
\hline & \multicolumn{2}{|c|}{ Group I A } & \multicolumn{2}{|c|}{ Group I B } & \multicolumn{2}{|c|}{ Group II } & \multicolumn{2}{|c|}{ Group III } & \multicolumn{2}{|c|}{ One way ANOVA } \\
\hline & Mean & SD & Mean & SI & Mean & SD & \begin{tabular}{|l|} 
Mean \\
\end{tabular} & SD & $\mathbf{F}$ & P value \\
\hline NLR & \multirow{2}{*}{$\begin{array}{c}2.94 \\
249.24\end{array}$} & 0.79 & 3.65 & 0.8 & $\begin{array}{ll}1.82 \\
\end{array}$ & 0.31 & $\begin{array}{ll}1.24 \\
\end{array}$ & 0.19 & 101.169 & $<0.001$ \\
\hline PLR & & 93.47 & 277.32 & 92.9 & 175.76 & 63.42 & 108.32 & 36.32 & 28.016 & $<0.001$ \\
\hline U.albumin & 20.50 & 16.61 & 102.92 & 26. & \begin{tabular}{|l|}
15.28 \\
\end{tabular} & 5.33 & \begin{tabular}{|l|}
5.28 \\
\end{tabular} & 2.15 & 265.809 & $<0.001$ \\
\hline U. creat & 0.13 & 0.07 & 0.23 & 0.0 & 0.08 & 0.03 & 0.08 & 0.01 & 58.726 & $<0.001$ \\
\hline \begin{tabular}{|l} 
UACR \\
\end{tabular} & 151.20 & 77.04 & 451.36 & 86.0 & \begin{tabular}{l|l|}
1 & 18.32 \\
\end{tabular} & 5.53 & 6.70 & 2.19 & 451.053 & $<0.001$ \\
\hline \multicolumn{11}{|c|}{ Post hoc test } \\
\hline & \multirow{2}{*}{\multicolumn{4}{|c|}{\begin{tabular}{|c|} 
Group IA VS Group IB \\
$<0.001$
\end{tabular}}} & \multirow{2}{*}{\multicolumn{4}{|c|}{$\frac{\text { Group IA VS Group II }}{<0.001}$}} & oup II VS & Group III \\
\hline NLR & & \multirow{2}{*}{\multicolumn{3}{|c|}{\begin{tabular}{c|}
$<0.001$ \\
0.177
\end{tabular}}} & & & & & \multicolumn{2}{|c|}{$<0.001$} \\
\hline \multirow{2}{*}{\multicolumn{2}{|c|}{$\frac{\text { PLK }}{\text { U.albumin }}$}} & & & & \multicolumn{4}{|c|}{$<0.001$} & \multicolumn{2}{|c|}{$<0.001$} \\
\hline & & \multicolumn{3}{|c|}{$<0.001$} & \multicolumn{3}{|c|}{0.153} & & \multicolumn{2}{|c|}{0.005} \\
\hline \multicolumn{2}{|c|}{ U. creat } & \multicolumn{3}{|c|}{$<0.001$} & \multirow{2}{*}{\multicolumn{3}{|c|}{0.001}} & & \multicolumn{2}{|c|}{0.901} \\
\hline UACR & & \multicolumn{2}{|c|}{$<0.001$} & & \multicolumn{2}{|r|}{$<0.001$} & & \multicolumn{3}{|c|}{$<0.001$} \\
\hline
\end{tabular}

Our study showed that there is high statistically significant increase as regard NLR, PLR and UACR in group IB when compared to group IA, II and III. In groupIA in comparison to group II In group II in comparison to group III.

Table (3): Correlation between NLR and PLR as regards all parameters in group I A

\begin{tabular}{|l|c|c|c|c|}
\hline \multirow{2}{*}{} & \multicolumn{2}{|c|}{ NLR } & \multicolumn{2}{c|}{ PLR } \\
\cline { 2 - 5 } & R & P value & R & P value \\
\hline PLR & 0.440 & 0.028 & & \\
\hline Age (Years) & 0.044 & 0.833 & -0.061 & 0.774 \\
\hline Weight (KG) & 0.073 & 0.729 & 0.069 & 0.744 \\
\hline Duration of disease (Years) & -0.178 & 0.404 & -0.270 & 0.202 \\
\hline Pulse (beat/mint) & 0.036 & 0.866 & 0.380 & 0.061 \\
\hline FBG & 0.292 & 0.157 & 0.197 & 0.346 \\
\hline PPG & -0.041 & 0.845 & 0.220 & 0.291 \\
\hline HBA1C & 0.662 & 0.001 & 0.432 & 0.031 \\
\hline WBCs & 0.002 & 0.991 & -0.469 & 0.018 \\
\hline HB & 0.109 & 0.605 & 0.242 & 0.244 \\
\hline PLT & -0.008 & 0.969 & 0.610 & 0.001 \\
\hline Neutrophil & 0.111 & 0.596 & -0.553 & 0.004 \\
\hline Lymphocyte & -0.532 & 0.006 & -0.778 & 0.001 \\
\hline U.albumin & 0.559 & 0.002 & 0.468 & 0.038 \\
\hline U.creat & -0.267 & 0.197 & -0.338 & 0.099 \\
\hline UACR & -0.097 & 0.004 & -0.066 & 0.025 \\
\hline e GFR & -0.328 & 0.109 & -0.263 & 0.204 \\
\hline S.Uric Acid & 0.055 & 0.794 & 0.294 & 0.154 \\
\hline S.Creatnine & 0.151 & 0.472 & 0.185 & 0.376 \\
\hline
\end{tabular}

Table (4): Correlation between NLR and PLR as regards all parameters in group I B.

\begin{tabular}{|l|c|c|c|c|}
\hline \multirow{2}{*}{} & \multicolumn{2}{|c|}{ NLR } & \multicolumn{2}{c|}{ PLR } \\
\cline { 2 - 5 } & R & P value & R & P value \\
\hline PLR & 0.725 & 0.001 & & \\
\hline Age (Years) & -0.070 & 0.741 & 0.172 & 0.410 \\
\hline Weight (KG) & 0.138 & 0.510 & -0.029 & 0.889 \\
\hline Duration of disease (Years) & 0.086 & 0.683 & 0.179 & 0.391 \\
\hline Pulse (beat/mint) & -0.111 & 0.598 & 0.071 & 0.735 \\
\hline FBG & 0.295 & 0.152 & 0.282 & 0.172 \\
\hline PPG & 0.126 & 0.549 & 0.003 & 0.988 \\
\hline HBA1C & 0.254 & 0.221 & 0.180 & 0.389 \\
\hline WBCs & -0.218 & 0.295 & -0.409 & 0.043 \\
\hline HB & 0.287 & 0.164 & 0.281 & 0.174 \\
\hline PLT & 0.183 & 0.382 & 0.579 & 0.002 \\
\hline Neutrophil & -0.086 & 0.683 & -0.425 & 0.034 \\
\hline Lymphocyte & -0.744 & 0.001 & -0.813 & 0.001 \\
\hline U.albumin & 0.579 & 0.002 & 0.733 & 0.001 \\
\hline U.creat & -0.259 & 0.211 & -0.177 & 0.398 \\
\hline UACR & -0.108 & 0.043 & -0.040 & 0.002 \\
\hline e GFR & 0.022 & 0.918 & -0.294 & 0.154 \\
\hline S.Uric Acid & -0.107 & 0.612 & -0.115 & 0.583 \\
\hline S.Creatnine & -0.074 & 0.725 & 0.200 & 0.338 \\
\hline
\end{tabular}


In our study, there is positive correlation between NLR, PLR, HBA1C and UACR in group IA and there is positive correlation between NLR and PLR and UACR in group IB. and there is positive correlation between NLR and UACR in group II. NLR also has negative correlation with Lymphocyte in group IA, IB and II. But there was no significant statistically correlation was found between NLR and other variables. In this study the ROC curve of NLR between group IA and group IB show cut off point 2.92 with sensitivity $64.00 \%$ and specificity $88.0 \%$ while ROC curve of NLR between group IA and group II show cut off point 2.2 with sensitivity $84 \%$ and specificity $98 \%$. While ROC curve of NLR between group II and group III show cut off point 1.5 with sensitivity $48 \%$ and specificity $98 \%$.

Table (5): Correlation between NLR and PLR as regards all parameters in group II.

\begin{tabular}{|l|c|c|c|c|}
\hline \multirow{2}{*}{} & \multicolumn{2}{|c|}{ NLR } & \multicolumn{2}{c|}{ PLR } \\
\cline { 2 - 5 } & R & P value & R & P value \\
\hline PLR & 0.240 & 0.094 & & \\
\hline Age (Years) & 0.108 & 0.453 & -0.183 & 0.204 \\
\hline Weight (KG) & 0.055 & 0.705 & -0.130 & 0.369 \\
\hline Duration of disease (Years) & -0.019 & 0.896 & 0.064 & 0.664 \\
\hline Pulse (beat/mint) & -0.129 & 0.371 & 0.177 & 0.220 \\
\hline FBG & -0.011 & 0.941 & 0.040 & 0.783 \\
\hline PPG & -0.225 & 0.117 & 0.027 & 0.850 \\
\hline HBA1C & -0.101 & 0.485 & -0.240 & 0.094 \\
\hline WBCs & 0.088 & 0.544 & -0.555 & 0.001 \\
\hline HB & -0.047 & 0.746 & 0.126 & 0.385 \\
\hline PLT & -0.002 & 0.989 & 0.542 & 0.001 \\
\hline Neutrophil & 0.270 & 0.058 & -0.593 & 0.001 \\
\hline Lymphocyte & -0.300 & 0.034 & -0.766 & 0.001 \\
\hline U.albumin & 0.542 & 0.003 & 0.351 & 0.022 \\
\hline U.creat & 0.202 & 0.160 & 0.134 & 0.353 \\
\hline UACR & -0.010 & 0.034 & -0.199 & 0.022 \\
\hline e GFR & 0.133 & 0.357 & 0.044 & 0.763 \\
\hline S.Uric Acid & -0.160 & 0.266 & 0.073 & 0.614 \\
\hline S.Creatnine & -0.091 & 0.531 & -0.134 & 0.352 \\
\hline \multicolumn{2}{|c|}{ Our study } & Also, there & \multicolumn{2}{c|}{ is } \\
\hline
\end{tabular}
correlation between PLR and PLT, HBA1C and UACR in group IA and there is positive correlation between PLR and PLT, Neutrophils and UACR in group IB and there is positive correlation between PLR and PLT and UACR in group II.

PLR also has negative correlation with Lymphocyte, neutrophil and WBC in group IA, IB and II respectively but there was no significant statistically correlation was found between PLR and other variables. Also, our results showed that the ROC curve of PLR between group IA and group IB show cut off point 288 with sensitivity $76.00 \%$ and specificity $48.0 \%$ while ROC curve of PLR between group IA and group II show cut off point 207 with sensitivity $72 \%$ and specificity $80 \%$. While ROC curve of PLR between group II and group III show cut off point 147 with sensitivity $68 \%$ and specificity $88 \%$.

Table (6): Statistical comparison between Group IA, IB, II and III as regards e GFR, uric acid and S. creatinine.

\begin{tabular}{|c|c|c|c|c|c|c|c|c|c|c|}
\hline & \multicolumn{2}{|c|}{ Group I A } & \multicolumn{2}{|c|}{ Group I B } & \multicolumn{2}{|c|}{ Group II } & \multicolumn{2}{|c|}{ Group III } & \multicolumn{2}{|c|}{$\begin{array}{l}\text { One way } \\
\text { ANOVA }\end{array}$} \\
\hline & Mean & SD & Mean & SD & Mean & SD & Mean & SD & $\mathbf{F}$ & \begin{tabular}{|l|} 
P value \\
\end{tabular} \\
\hline e GFR & 95.75 & 21.38 & 60.21 & 18.03 & 97.74 & 17.11 & 104.80 & 13.47 & 33.671 & $<0.001$ \\
\hline S.Uric Acid & 4.32 & 1.03 & 5.40 & 1.28 & 4.10 & 0.83 & 3.45 & 0.80 & 17.853 & $<0.001$ \\
\hline S.Creatnine & 0.98 & 0.24 & 1.43 & 0.42 & \begin{tabular}{|l|}
0.94 \\
\end{tabular} & 0.17 & 0.89 & 0.09 & 27.606 & $<0.001$ \\
\hline \multicolumn{11}{|c|}{ Post hoc test } \\
\hline & \multicolumn{3}{|c|}{ Group IA VS Group IB } & \multicolumn{4}{|c|}{ Group IA VS Group II } & \multicolumn{3}{|c|}{ Group II VS Group II } \\
\hline e GFR & \multicolumn{3}{|c|}{$<0.001$} & \multirow{2}{*}{\multicolumn{4}{|c|}{0.645}} & \multirow{2}{*}{\multicolumn{3}{|c|}{\begin{tabular}{|l|}
0.104 \\
\end{tabular}}} \\
\hline $\begin{array}{l}\text { S.Uric Acid } \\
\end{array}$ & \multicolumn{3}{|c|}{$<0.001$} & \multirow{2}{*}{\multicolumn{4}{|c|}{0.340}} & \multicolumn{2}{|c|}{0.007} & \\
\hline S.Creatnine & \multicolumn{3}{|c|}{$<0.001$} & & & & & \multicolumn{3}{|c|}{0.426} \\
\hline
\end{tabular}

In our study, eGFR were found to be significantly lower in group IB when compared to group IA but there is no statistically significant difference between otherS.creatinine and S.Uric acid were found to be significantly higher in group IB when compared group IA, but there is no statistically significant difference between other groups as regard S.creatinine and S.Uric acid.

Table (7): Statistical comparison between Group IA, IB, II and III as regards history.

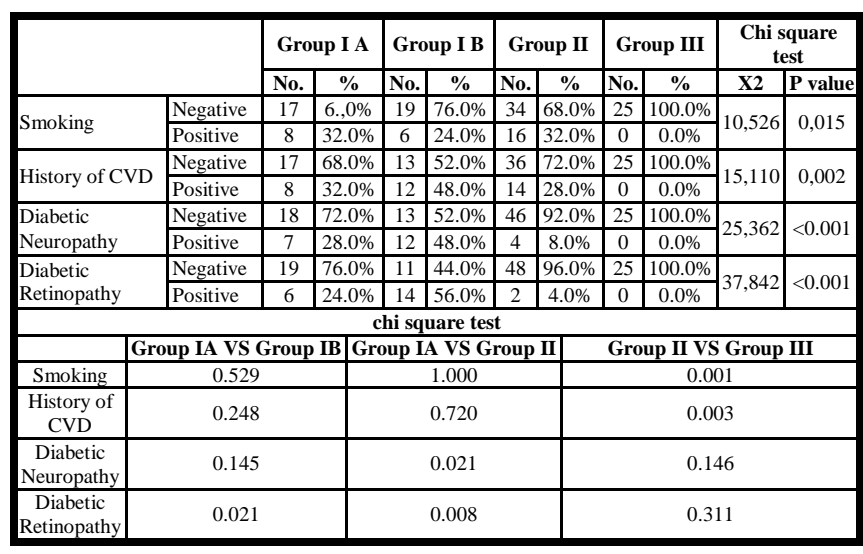

In our study there is statistically significant increase as regard history of smoking in group II in comparison to group III. Also our results showed that there is statistically significant increase as regard history of CVD in group II in comparison to group III in the form of ECG changes and anginal pain.

In our study there is high statistically significant increase as regard history of diabetic neuropathy in group IA in comparison to group II.In our study there is high statistically significant increase as regard history of Diabetic retinopathy in group IA when compared to group II and in group IB when compared to group IA. 
Our study showed that there is high statistically significant increase as regard duration of disease of diabetes in group IA when compared to group II and in group IB when compared to group IA. Also our study finds that that there is high statistically significant increase as regard kidney Ultrasound (increase nephropathy) in group IA when compared to group II and in group IB when compared to group IA. Our study showed that there is high statistically significant increase as regard urine analysis finding (protein and casts) in group IB when compared to group IA.

Table (8): Comparison between Group IA, IB, II and III as regards FBG, PPG and HBA1C.

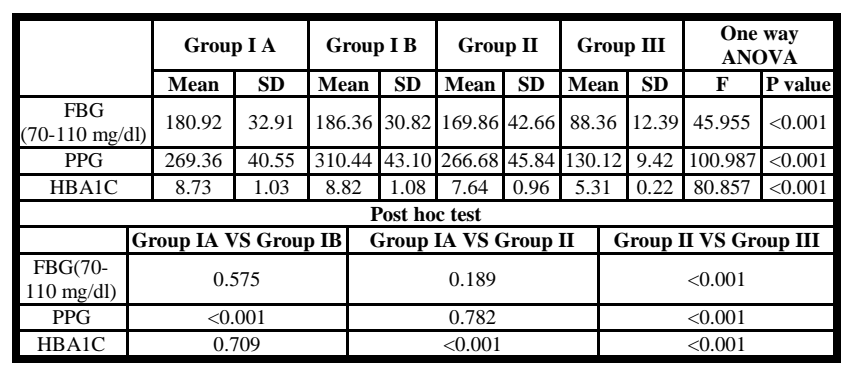

Also, our result shows that there was statistically significant increase as regard PPG in group IB in comparison to group IA,and there is statistically significant increase in group IA in comparison to group II, as regard HBA1C. There is no statistically significant deference between group IA and group II as regard FBG and PPG.

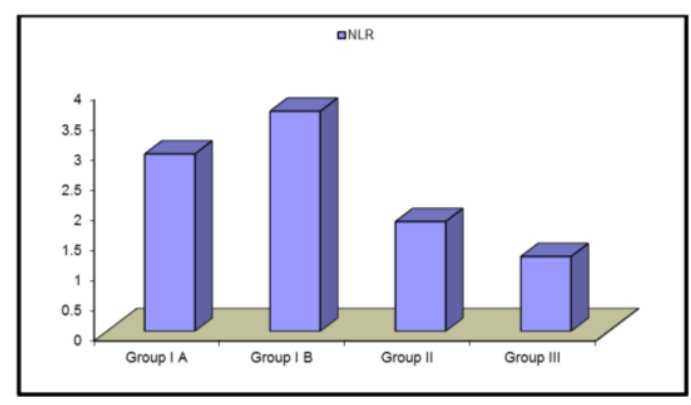

Figure (1): NLR regarding studied groups.

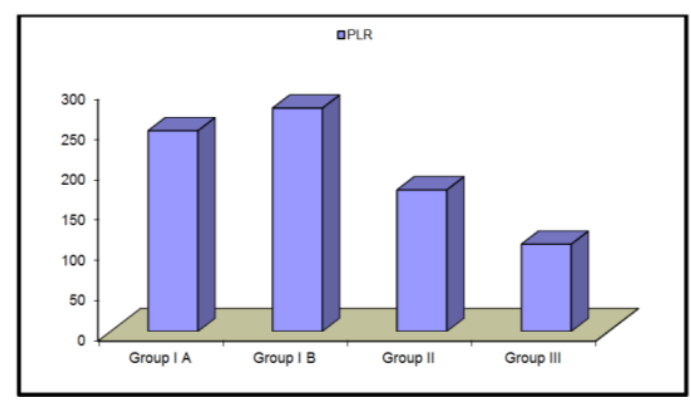

Figure: (2): PLR regarding studied groups.

\section{DISCUSSION}

Our study showed that there is high statistically significant increase as regard neutrophils count $/ \mathrm{mm} 3$ in group IB in comparison to group IA, II and III. There was statistically significant decrease as regard Lymphocytes count $/ \mathrm{mm} 3$ in group IB in comparison to group IA, II and III. And in group IA in comparison to group II and III.In group II in comparison to group III.

Goldberg ${ }^{(11)}$ showed thatmultiple studies have established that inflammatory markers such as neutrophilia and relative lymphocytopenia are independent markers of many diseases, especially complications of DM, such as DN. There is statistically significant increase as regard Platelets count $/ \mathrm{mm} 3$ in group IB in comparison to group IA, II and III. and in group IA in comparison to group II and III.

Our study showed that there is high statistically significant increase as regard NLR, PLR and UACR in group IB when compared to group IA, II and III. and in group IA in comparison to group II and in group II in comparison to group III.

Neutrophil lymphocyte ratio (NLR) has been evaluated and used as inflammatory marker in malignancies, infection and coronary artery diseases. The use of NLR as inflammatory marker may be simple and readily available biomarkers ${ }^{(12)}$.

PLR is novel inflammatory biomarkers used as prognostic factors in various diseases

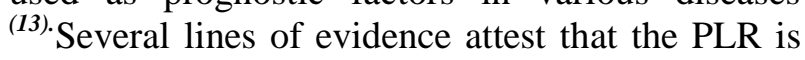
associated with outcomes of patients with ankylosing spondylitis, non-small cell lung cancer, and acute coronary syndrome ${ }^{(14) .}$

In our study, there is positive correlation between NLR, PLR, HBA1C and UACR in group IA and there is positive correlation between NLR and PLR and UACR in group IB. and there is positive correlation between NLR and UACR in group II. NLR also has negative correlation with Lymphocyte in group IA, IB and II. But there was no significant statistically correlation was found between NLR and other variables.

Shiny et al. ${ }^{(15)}$ have shown that NLR is correlated with increasing severity of glucose intolerance and insulin resistance and can be used as a prognostic marker for macro- and microvascular complications in patients with glucose intolerance. 
In this study the ROC curve of NLR between group IA and group IB show cut off point 2.92 with sensitivity $64.00 \%$ and specificity $88.0 \%$ while ROC curve of NLR between group IA and group II show cut off point 2.2 with sensitivity $84 \%$ and specificity 98\%. While ROC curve of NLR between group II and group III show cut off point 1.5 with sensitivity $48 \%$ and specificity $98 \%$.Similar to our results Huang et al $^{(16)}$ have also found that NLR values were significantly higher in diabetic patients with evidence of nephropathy $(2.48 \pm 0.59)$ than in diabetic patients without nephropathy $(2.20 \pm 0.62)$.

Akbas et al. ${ }^{(17)}$ have shown that NLR was significantly elevated in patients with increased albuminuria pointing toward a relationship between inflammation and endothelial dysfunction in diabetics with nephropathy.

In agreement with our study Sagar et al. ${ }^{(10)}$ found that there was a significant correlation between NLR and DN, implying that inflammation and endothelial dysfunction could be an integral part of DN. An association between NLR and worsening renal function in diabetic patients has been determined ${ }^{(18) .}$

In our study, there is positive correlation between PLR and PLT, HBA1C and UACR in group IA and there is positive correlation between PLR and PLT, Neutrophils and UACR in group IB and there is positive correlation between PLR and PLT and UACR in group II. PLR also has negative correlation with Lymphocyte, neutrophil and WBC in group IA, IB and II respectively but there was no significant statistically correlation was found between PLR and other variables. Also, our results showed that the ROC curve of PLR between group IA and group IB show cut off point 288 with sensitivity $76.00 \%$ and specificity $48.0 \%$ while ROC curve of PLR between group IA and group II show cut off point 207 with sensitivity $72 \%$ and specificity $80 \%$. While, ROC curve of PLR between group II and group III show cut off point 147 with sensitivity $68 \%$ and specificity $88 \%$.In agreement with our results Demirtas et al. ${ }^{(19)}$ confirmed that PLR levels were found to be independent predictor of diabetes and to be independent predictor of impaired glucose regulation in diabetic patients.

Descamps-Latscha et al. ${ }^{(20)}$ found that there was a positive correlation between PLR and NLR IL-6 and TNF- $\alpha$ in ESRD patients. Moreover, patients with higher PLR had higher NLR, IL-6, and TNF- $\alpha$ level.
In our study, eGFR were found to be significantly lower in group IB when compared to group IA but there is no statistically significant difference between other groups as regard eGFR.

In agreement with our results Sagar et al. ${ }^{(10)}$ find that there was significant difference among the groups in respect to eGFR values. Patients with albuminuria had significantly low eGFR as compared to those patients with normal albumin levels.

In our study there is statistically significant increase as regard history of smoking in group II in comparison to group III. Numerous studies have established that smoking promotes onset and progress at all stages of DN in T1DM and T2DM ${ }^{(21)}$.Also our results showed that there is statistically significant increase as regard history of CVD in group II in comparison to group III in the form of ECG changes and anginal pain. Vinik et al. ${ }^{(22)}$ find that a three-fold increase in the incidence of CVD in DM patients has been reported and CVD has become the major risk factor for DM-associated morbidity and mortality.

In our study there is high statistically significant increase as regard history of diabetic neuropathy in group IA in comparison to group II. Liu et al. ${ }^{(23)}$ study showed that T2DM patients with higher NLR levels might be more likely to develop peripheral neuropathy complication..

In our study there is high statistically significant increase as regard history of Diabetic retinopathy in group IA when compared to group II and in group IB when compared to group IA. In agreement with our study Moursy et al. ${ }^{(24)}$ showed that NLR values were significantly higher in diabetic patients with retinopathy, neuropathy and nephropathy than those of diabetic patients without any microvascular complications and healthy controls.

Our study showed that there is high statistically significant increase as regard urine analysis finding (protein and casts) in group IB when compared to group IA. Also, our result shows that there was statistically significant increase as regard PPG in group IB in comparison to group IA, and there is statistically significant increase in group IA in comparison to group II, as regard HBA1C. There is no statistically significant deference between group IA and group II as regard FBG and PPG.

In agreement with our results Hussain et $a l^{(25)}$ find that Increased NLR level is associated with 
elevated HBA1c and poor glycemic control in patients of type 2 diabetes mellitus. It can be used as a disease monitoring tool during the follow up of diabetic patients.

\section{CONCLUSION}

NLR \& PLR was significantly associated with DN, and high NLR \& PLR values may be considered as a predictor and a prognostic risk marker of DN. NLR \& PLR is an easy to calculate parameters in the laboratory. These tests are simple, inexpensive, and done routinely. They can be an alternative for other costlier inflammatory markers such as ILs, TNF, and cytokines.

\section{REFERENCES}

1- Smith RJ (2016): Endocrine Disease and Metabolic Disease, Sector $\mathrm{X}$, Diabetes Mellitus, and Hypoglycemia. sited in Cecil Essentials of Medicine 9th Edition, 657-675.

2- Tuttle KR, Bakris GL, Bilous RW et al. (2017): Diabetic Kidney Disease Sited in American Diabetes Association (ADA) Microvascular complications and foot care. Sec, 10, In Standards of Medical Care in Diabetes. Diabetes Care, 40(1): S88-S98:95-104.

3- Perkins BA, Ficociello LH, Roshan B et al. (2010): In patients with type 1 diabetes and new onset microalbuminuria at the development of advanced chronic kidney disease may not require progression to proteinuria. Kidney Int., 77:57-64.

4- De Boer IH, Rue TC, Hall YN et al. (2011): Temporal trends in the prevalence of diabetic kidney disease in the United States. JAMA., 305:2532-2539.

5- Rudiger A, Burckhardt OA, Harpes $P$ et al. (2006): The relative lymphocyte count on hospital admission is a risk factor for long term mortality in patients with acute heart failure. Am J EmergMed;.,24:4514.

6- Amaylia OE, Hendarsyah S, Sumartini D et al. (2013): The Role of Neutrophil Lymphocyte Count Ratio as an Inflammatory Marker in Systemic Lupus Erythematosus, The Indonesian Journal of Internal Medicine. 170-174.

7- Celikbilek M, Dogan S, Ozbakır O et al. (2013): Neutrophil-lymphocyte ratio as a predictor of disease severity in ulcerative colitis. J Clin Lab Anal., 27:72-76.
8- Núñez J, Núñez E, Bodí $V$ et al. (2008); Heatta AM, Llàcer A Am J Cardiol., 101(6):747-52.

9- Turkmen K, ErdurFM, Ozcicek $\mathbf{F}$ et al. (2013): Platelet-to-lymphocyte ratio better predicts inflammation than neutrophil-tolymphocyte ratio in end-stage renal disease patients. Hemodial Int., 17:391-396.

10-Sagar A, Sachin C, Nitin N et al. (2017): Study of Neutrophil-lymphocyte Ratio as Novel Marker for Diabetic Nephropathy in Type 2 Diabetes, Indian Journal of Endocrinology and Metabolism, 387-392.

11- Goldberg RB (2009): Cytokine and cytokinelike inflammation markers, endothelial dysfunction, and imbalanced coagulation in development of diabetes and its complications. J Clin Endocrinol Metab., 94:3171-82.

12- Chua W, Charles KA, Baracos VE et al. (2011): Neutrophil-lymphocyte ratio predicts chemotherapy outcomes in patients with advanced colorectal cancer $\mathrm{Br} \mathrm{J}$ Cancer, 104(8):1288-1295.

13- Yıldız E, Yavuzcan A, Ustün Y et al. (2013): Evaluation of mean platelet volume, neutrophil/lymphocyte ratio and platelet/ lymphocyte ratio in advanced stage endometriosis with endometrioma. J Turk Ger Gynecol Assoc., 14:210-5

14- Akkaya E, Gul M, Ugur M et al. (2014): Platelet to lymphocyte ratio: A simple and valuable prognostic marker for acute coronary syndrome, Int J Cardiol., 28:13-15.

15-Shiny A, Bibin YS, Shanthirani CS et al. (2014): Association of neutrophil-lymphocyte ratio with glucose intolerance: An indicator of systemic inflammation in patients with type 2 diabetes. Diabetes Technol There.,16:524 30.

16- Huang W, Huang J, Liu Q et al. (2015): Neutrophil-lymphocyte ratio is a reliable predictive marker for early-stage diabetic nephropathy. Clin Endocrinol (Oxf), 82:229-33.

17- Akbas EM, Demirtas L, Ozcicek A et al. (2014): Association of epicardial adipose tissue, neutrophil-to-lymphocyte ratio and platelet-tolymphocyte ratio with diabetic nephropathy. Int $\mathrm{J}$ Clin Exp Med.,7:1794-801. 
18- Azab B, Shah N, Akerman $M$ et al. (2013): Value of platelet/lymphocyte ratio as a predictor of all-cause mortality after non-ST-elevation myocardial infarction. J Thrombolysis, 34:326334.

19- Demirtas L, Degirmenci H, Akbas EM et al. (2015): Association of hematological indicies with diabetes, impaired glucose regulation and microvascular complications of diabetes. International Journal of Clinical and Experimental Medicine,8(7):11420-11427.

20- Descamps-Latscha B, Herbelin A, Nguyen AT et al. (1995): Balance between IL-1 beta, TNFalpha, and their specific inhibitors in chronic renal failure and maintenance dialysis. Relationships with activation markers of $\mathrm{T}$ cells, $\mathrm{B}$ cells, and monocytes. J Immunol, 154:882-892.

21-Orth SR (2002): Smoking and the kidney. Journal of the American Society of Nephrology, 13, 1663-1672.
22- Vinik AI, Erbas T, Casellini CM (2013): Diabetic cardiac autonomic neuropathy, inflammation and cardiovascular disease. $\mathbf{J}$ Diabetes Investig., 2013;4:4-18.

23- Liu S, Zheng H., Xiaoming $Z$ et al. (2017): Neutrophil-to-lymphocyte ratio is associated with diabetic peripheral neuropathy in type 2 diabetes patients. Diabetes Research and Clinical Practice, 130: 90 - 97.

24- Moursy EY, Megallaa MH, Mouftah RF et al. (2015): Relationship between neutrophil lymphocyte ratio and microvascular complications in Egyptian patients with type 2 diabetes. Am J Intern Med., 2015;3:250-5.

25-Hussain M, Babar MZM, Akhtar L et al. (2017): Neutrophil lymphocyte ratio (NLR): A well assessment tool of glycemic control in type 2 diabetic patients. Pakistan Journal of Medical Sciences, 33(6):1366-1370. 